Phosphat i dyl ser i ne- medi at ed phagocyt osi s of ant i cancer drug-treat ed cel Is by macrophages

\begin{tabular}{|l|l|}
\hline 著者 & Shi rat suchi Aki ko, Nakani shi Yoshi nobu \\
\hline $\begin{array}{l}\mathrm{j} \text { our nal or } \\
\text { publ i cat i on ti l l e }\end{array}$ & Journal of Bi ochem st ry \\
\hline vol une & 126 \\
\hline number & 6 \\
\hline page r ange & $1101-1106$ \\
\hline year & 1999- 01-01 \\
\hline URL & ht t p: //hdl . handl e. net /2297/14557 \\
\hline
\end{tabular}




\title{
Phosphatidylserine-Mediated Phagocytosis of Anticancer Drug-Treated Cells by Macrophages ${ }^{1}$
}

\author{
Akiko Shiratsuchi and Yoshinobu Nakanishi ${ }^{2}$ \\ Faculty of Pharmaceutical Sciences, Kanazawa University, 13-1 Takara-machi, Kanazawa, Ishikawa 920-0934
}

Received July 19, 1999; accepted September 29, 1999

\begin{abstract}
Apoptotic cells are rapidly phagocytosed and eliminated from the organism. Although cancer cells apoptose when treated with anticancer drugs, how those cells are recognized by phagocytic cells has remained unclear. The human leukemia cell line Jurkat was cultured with doxorubicin or bufalin and induced to undergo apoptosis accompanied by phosphatidylserine externalization. When apoptotic Jurkat cells were mixed with mouse peritoneal macrophages, efficient phagocytosis was observed. Apoptosis and phagocytosis of Jurkat cells were both inhibited by Z-VAD-FMK, and phagocytosis was significantly reduced in the presence of phosphatidylserine-containing liposomes. These results suggest that anticancer drugs induce apoptosis-dependent and phosphatidylserine-mediated phagocytosis in cancer cells.
\end{abstract}

Key words: anticancer drug, apoptosis, phagocytosis, phosphatidylserine.

Apoptotic cells are engulfed by phagocytic cells, and this event has been presumed to prevent damage to surrounding tissues by the noxious contents of dead cells (reviewed in Refs. 1, 2). Another important role of phagocytosis of apoptotic cells has recently been proposed: that is, phagocytic cells present antigens, which apoptotic cells contain, on their surface and stimulate $\mathrm{T}$ lymphocytes (3-7). Furthermore, injection of macrophages that had phagocytosed apoptotic tumor cells led to tumor regression in rats $(7)$. These observations strongly suggest that apoptotic cell phagocytosis occurs to eliminate unwanted cells not only directly but also indirectly by activating the immune response. It is important to elucidate the mechanism by which phagocytic cells recognize and phagocytose apoptotic cells in order to apply this phenomenon to the development of novel therapeutics against cancer.

Phagocytes should distinguish target apoptosing cells from living cells to selectively engulf the former. A variety of molecules, such as proteins, sugars, and phospholipids, have been suggested to act as a "phagocytosis marker," which is exposed on the surface of apoptotic cells and recognized by phagocytes (reviewed in Refs. 8-10). Among them, the membrane phospholipid PS has been most

\footnotetext{
${ }^{1}$ This work was supported by a Grant-in-Aid for Scientific Research from the Ministry of Education, Science, Sports and Culture of Japan, a grant from the Organized Research Combination System of the Science and Technology Agency of Japan, a grant from the Hokuriku Industrial Advancement Center, and a grant from the ONO Medical Research Foundation.

2 To whom correspondence should be addressed. Phone: +81-76-2344481, Fax: +81-76-234-4480, E-mail: nakanaka $G$ kenroku.kana2awa-u.ac.jp

Abbreviations: FITC, fluorescein isothiocyanate; PBS, phosphatebuffered saline; PC, phosphatidylcholine; PS, phosphatidylserine; Z-VAD-FMK, benzyloxycarbonyl-Val-Ala-Asp (OMe) fluoromethylketone.
}

C 1999 by The Japanese Biochemical Society. extensively characterized (reviewed in Ref. 11). In many apoptosing cells, including lymphocytes (12), vascular smooth muscle cells (13), vascular endothelial cells (14), spermatogenic cells (15), and Fas-overexpressing HeLa cells (16), externalized PS serves as a marker to allow phagocytes to recognize target cells. Many anticancer drugs induce apoptosis in cancer cells (reviewed in Refs. 17-19), and this is often accompanied by PS externalization (20). Apoptotic cells with externalized PS are, however, not necessarily phagocytosed in the PS-mediated manner (21, 22). We investigated here how anticancer drug-treated apoptotic cancer cells are phagocytosed by macrophages.

\section{MATERIALS AND METHODS}

Cell Culture and Apoptosis Analysis-Jurkat cells, a human leukemia T-cell line, were grown in RPMI 1640 (Nissui Pharmaceutical, Tokyo) containing 10\% fetal bovine serum at $37^{\circ} \mathrm{C}$ in a humidified atmosphere with $5 \%$ $\mathrm{CO}_{2}$ in air. The cells were treated with either doxorubicin (Sigma, MO) $(0.3 \mu \mathrm{g} / \mathrm{ml}$ ) or bufalin (provided by $\mathrm{K}$. Nakaya) $\left(10^{-7} \mathrm{M}\right)$. Cell viability and chromatin condensation were analyzed under a microscope after staining cells with trypan blue or Hoechst 33342 , respectively. PS externalization was determined by flow cytometry (EPICS$\mathrm{XL}$; Coulter, FL) as described previously $(23,24)$. Briefly, test cells were treated with FITC-labeled annexin V (Bender MedSystems, Vienna, Austria) and propidium iodide, and cells that were less intensely stained with propidium iodide were gated and analyzed for the binding of FITC. annexin V. For confirmation of cell surface binding of FTTC-annexin V, cells mixed with the fluorescent dyes were further treated with trypan blue $(2 \mathrm{mg} / \mathrm{ml})$ at $\mathrm{pH} 4.5$ prior to flow cytometry. Trypan blue, a plasma membrane nonpermeable dye, binds to FITC and diminishes its fluorescence because of energy transfer (quenching) (25). To inhibit apoptosis, the caspase inhibitor Z.VAD-FMK 
(26) (Peptide Institute, Osaka) was added to cultures $1 \mathrm{~h}$ before anticancer drug treatment.

Macrophage Preparation and Phagocytosis AssayMacrophages were prepared from peritoneal fluids of thioglycollate-inoculated $\mathrm{BDF}_{1}$ mice (female, 8 weeks old) as described previously (16) and cultivated on cover slips (15 mm diameter) in RPMI 1640 containing 10\% fetal bovine serum at $37^{\circ} \mathrm{C}$ until use. The phagocytosis assay was performed essentially as described (15). Briefly, target cells were labeled with biotin (NHS-LS-Biotin; Pierce, Rockford, IL), mixed with macrophages (at a ratio of 10 target cells to one macrophage), and incubated at $37^{\circ} \mathrm{C}$ for $2-3 \mathrm{~h}$. The mixture was treated with trypsin $(0.5 \mathrm{mg} / \mathrm{ml})$ to remove Jurkat cells free from or lightly attaching to macrophages. The remaining cells were further treated with PBS containing paraformaldehyde (2\%), glutaraldehyde (0.5\%), and Triton X-100 (0.05\%), then supplemented with FITC-conjugated avidin (fluorescein-avidin D; Vector Laboratories, Burlingame, CA). To analyze chromatin condensation and nuclear morphology of phagocytosed Jurkat cells, the fixed cells were further treated with propidium iodide, which binds to DNA. The number of macrophages containing engulfed cells was determined under a fluorescence/phase-contrast microscope and expressed relative to that of total macrophages, i.e., as the phagocytic index. The mean and SD of a typical example from at least three independent experiments are presented. For liposome preparations, phospholipids (Avanti Polar Lipids, Alabaster, AL) were dried as films, suspended in PBS, and sonicated. The liposomes were composed of either
PC only (PC-liposomes) or a combination of PC and PS at a molar ratio of $7: 3$ (PS-liposomes).

\section{RESULTS}

Apoptosis of Anticancer Drug-Treated Jurkat CellsWe chose two apoptosis-inducing anticancer drugs, doxorubicin and bufalin. The drugs possess distinct modes of action: doxorubicin intercalates DNA and mainly affects nucleic acid synthesis (27), while bufalin inhibits $\mathrm{Na}^{+}, \mathrm{K}^{+}$. ATPase in the plasma membrane (28). Human leukemic Jurkat cells were cultured in the presence of these drugs, and cell viability and chromatin condensation were examined (Fig. 1A). Both drugs showed similar effects: cell viability decreased (left panel) and the number of cells with condensed chromatin increased (right panel) as the time of drug treatment was prolonged. Chromatin condensation caused by either drug was significantly inhibited in the presence of a caspase inhibitor, Z-VAD-FMK (Fig. 1B). These results indicated that doxorubicin and bufalin induce apoptosis in Jurkat cells.

We next examined whether this apoptosis is accompanied by PS externalization. To do so, drug-treated Jurkat cells were stained with FTTC-annexin V and propidium iodide, and analyzed by flow cytometry. When the cells less intensely stained with propidium iodide were examined for the binding of FITC-annexin V, ati extra peak with more fluorescence appeared after treatment with either drug, although the size of the peaks was different (left panels in Fig. 2A). Such peaks completely disappeared when the cells
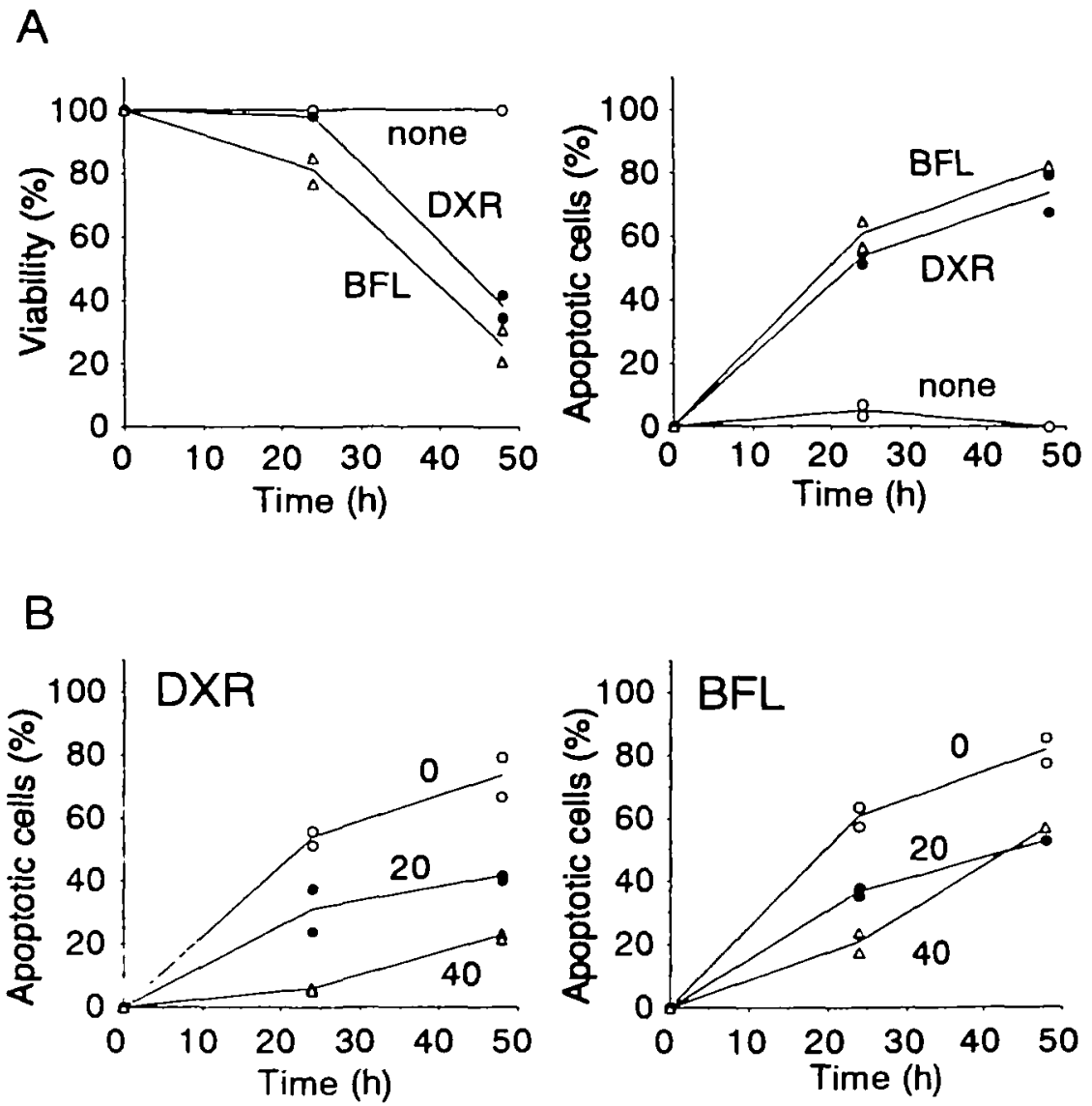

Fig. 1. Anticancer drug-induced apoptosis of Jurkat cells. (A) Jurkat cells treated with doxorubicin (DXR) or bufalin (BFL) for the indicated periods were analyzed for cell viability (trypan blue staining) (left) and occurrence of apoptosis (chromatin condensation) (right). (B) Jurkat cells were incubated with Z-VAD-FMK (20 or $40 \mu \mathrm{M}$ ) for $1 \mathrm{~h}$ prior to the treatment with doxorubicin (left) or bufalin (right), and the extent of apoptosis was determined. 

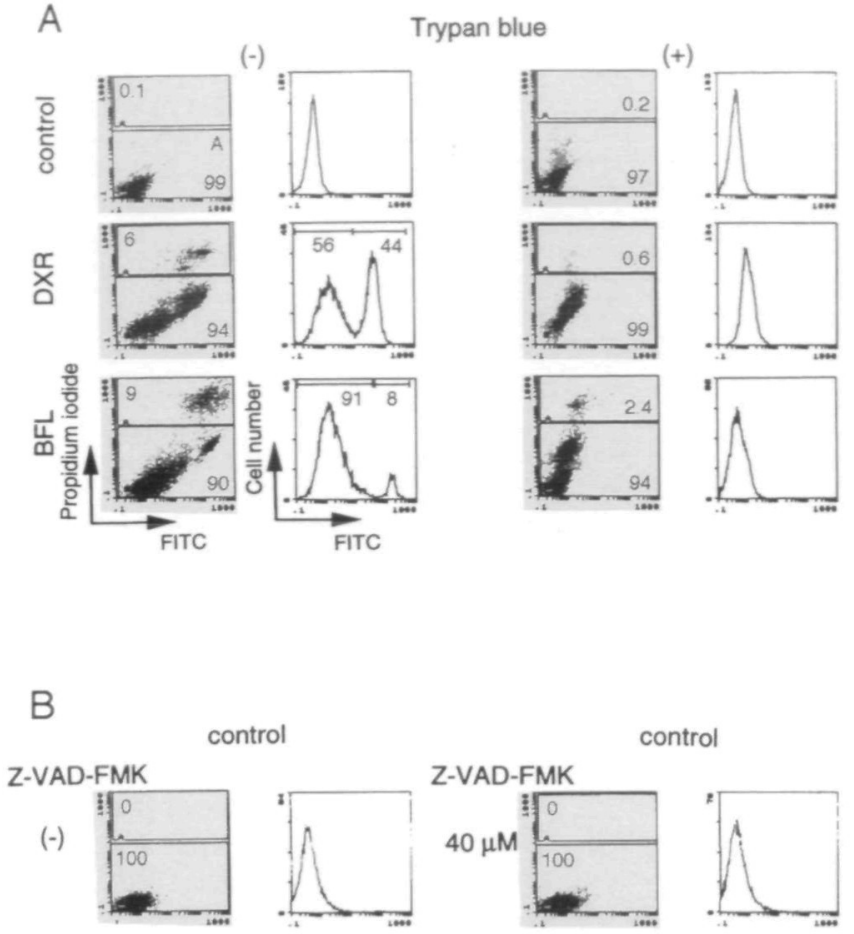

control
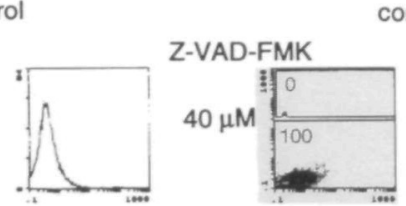

control

DXR
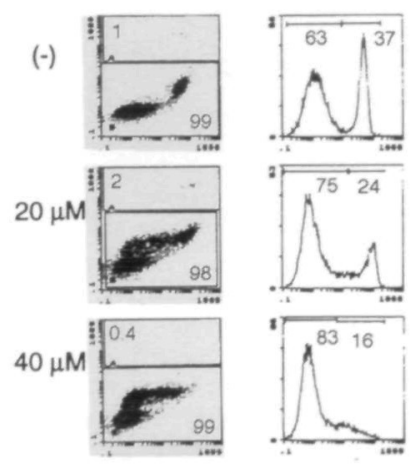

Fig. 2. PS externalization in anticancer drug-treated Jurkat cells. (A) Jurkat cells that had been treated with doxorubicin or bufalin for $24 \mathrm{~h}$ were analyzed for PS externalization by flow cytometry in the absence $(-)$ or presence $(+)$ of trypan blue. Cells less intensely stained with propidium iodide (zone $A$ in the left-most panel) were analyzed for the binding of annexin V (histograms at the right). The numbers indicate the percentages of cells in the corresponding areas. (B) The same assay was conducted with cells that were cultured in the presence of Z-VAD-FMK (20 or $40 \mu \mathrm{M}$ ).

were treated with trypan blue to quench fluorescence from the cell surface (right panels), indicating that cells corresponding to the extra peaks contained surface-bound FTTC. annexin V. Moreover, the addition of Z-VAD-FMK inhibited the appearance of such peaks (Fig. 2B). From these results, we concluded that $\mathrm{PS}$, which is normally restricted to the cytoplasmic side of living cells, was externalized and exposed on the surface of apoptotic Jurkat cells upon treatment with either doxorubicin or bufalin.

Phagocytosis of Anticancer Drug-Treated Jurkat Cells by Mouse Peritoneal Macrophages-Jurkat cells that had been cultured with the drugs for $24 \mathrm{~h}$ were labeled with biotin and mixed with mouse peritoneal macrophages. The mixture was incubated for $2-3 \mathrm{~h}$, washed, permealized, and supplemented with FITC-avidin. When the reacted cells were examined under a fluorescence/phase-contrast microscope, many fluorescent particles were detectable in the cytoplasm of macrophages (Fig. 3A). Jurkat cells left untreated with the drugs were not significantly engulfed by macrophages (Fig. 3B). There was no significant difference between the two drugs. When the macrophages that reacted with doxorubicin-treated Jurkat cells were further stained with propidium iodide, many phagocytosed Jurkat cells whose nuclei were not intensely stained or fragmented were observed (Fig. 3C). A similar result was obtained with bufalin-treated Jurkat cells (data not shown). These results indicated that macrophages phagocytosed drug-treated Jurkat cells before their chromatin condensed and nuclei were fragmented. Quantification of the phagocytosis reaction revealed that Jurkat cells treated with either drug became susceptible to phagocytosis by macrophages (Fig. 3D). Phagocytosis was significantly reduced when Jurkat cells were treated with the anticancer drugs in the presence of Z-VAD-FMK (Fig. 3E), indicating that phagocytosis was dependent on apoptosis. The above results all showed that Jurkat cells treated with either doxorubicin or bufalin were phagocytosed by peritoneal macrophages at early stages of apoptosis.

Involvement of PS in Phagocytosis of Anticancer DrugTreated Cells-Since externalized PS often serves as a marker in phagocytosis of apoptotic cells (11), we next asked if this is the case with anticancer drug-treated Jurkat cells, by conducting the phagocytosis assay in the presence of liposomes. Liposomes containing PS and PC significantly inhibited phagocytosis of Jurkat cells treated with either drug, whereas those composed of only PC showed a minimal effect (Fig. 4). These results suggested that phagocytosis of the drug-treated Jurkat cells by macrophages was mediated, at least partly, by PS exposed on the cell surface.

Significant levels of phagocytosis, however, always occurred in the presence of a maximal amount of PS-liposomes, suggesting the existence of PS-independent phagocytosis. We thus investigated the involvement of other possible phagocytosis markers (Fig. 5). A synthetic peptide containing the integrin-binding sequence RGDS; fucoidan and dextran sulfate, specific inhibitors of class $A$ scavenger receptor types I and $I$; and $N$-acetyl-D-glucosamine, a ligand for lectin-like receptors, all showed some inhibition, but were much less effective than PS-liposomes. Furthermore, simultaneous addition of PS-liposomes and fucoidan or dextran sulfate gave no additive effect. Similar results were obtained in the phagocytosis assays of Jurkat cells treated with either doxorubicin or bufalin. These results suggest that as-yet unidentified molecules, in addition to PS, are involved in the recognition by macrophages of apoptotic Jurkat cells.

\section{DISCUSSION}

Although anticancer drugs induce apoptosis that is accompanied by externalization of PS in many cancer cell lines (20), it has not yet been shown whether those cells undergo PS-mediated phagocytosis. The present study showed that macrophages phagocytosed Jurkat cells, which were undergoing apoptosis induced by treatment with either doxorubicin or bufalin, in a manner inhibitable by PS-containing 
A

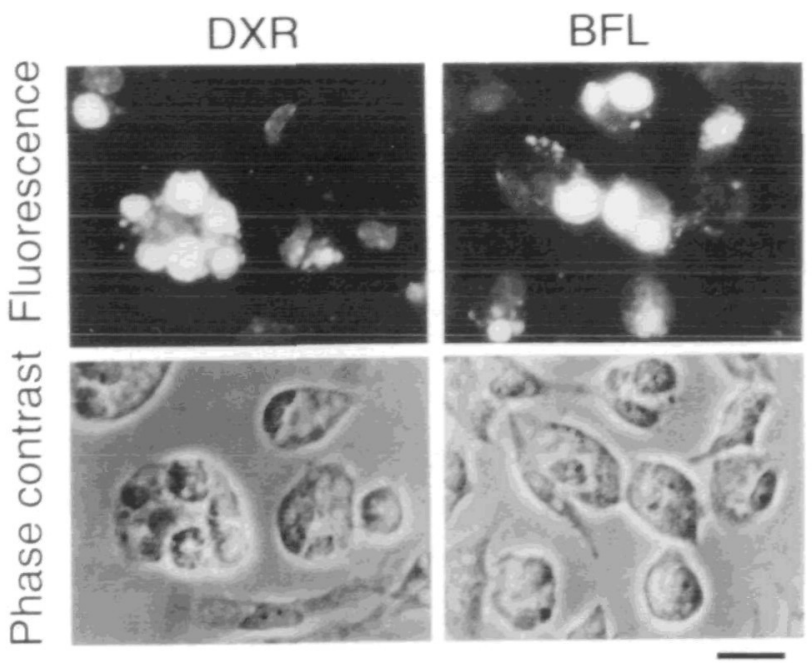

R
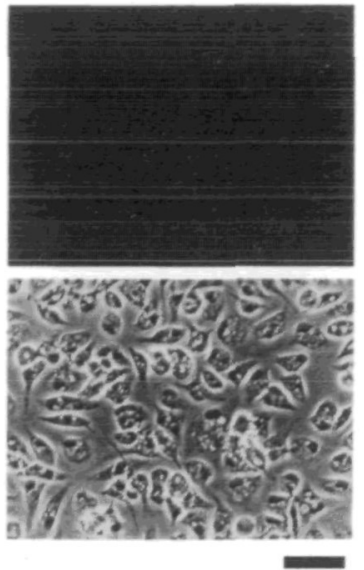

C
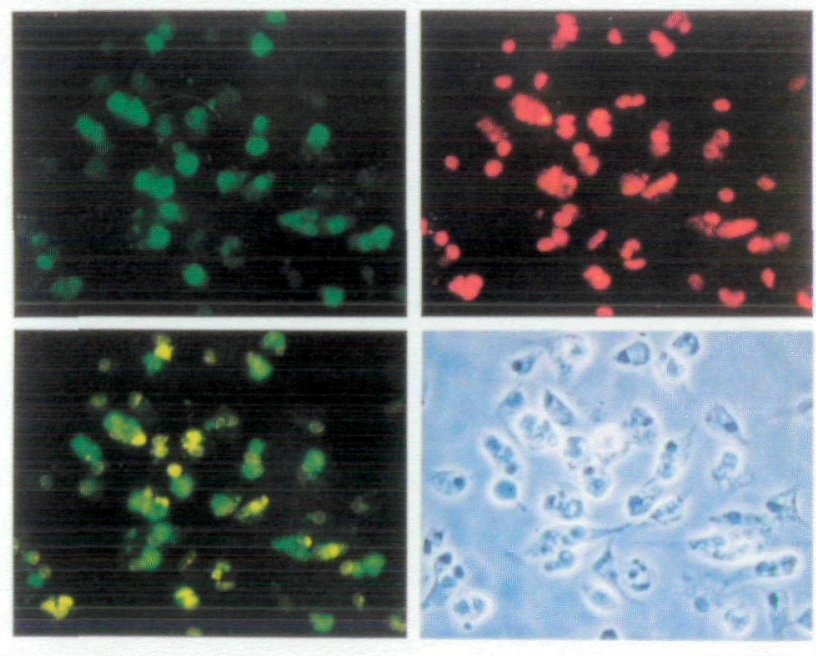

D

E

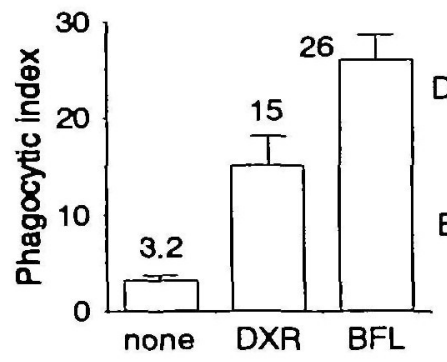

Z-VADFMK

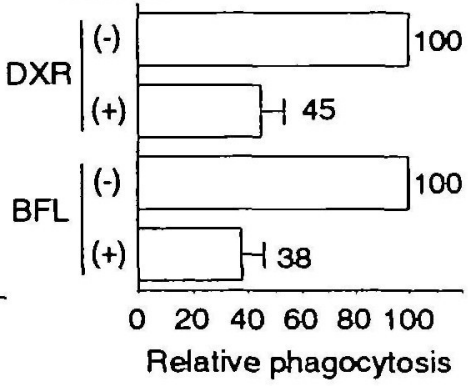

Fig. 3. Phagocytosis of antican. cer drug-treated Jurkat cells by mouse peritoneal macrophages. (A) The mixture of Jurkat cells and macrophages after the phagocytosis reaction was examined under a fluorescence/phase-contrast microscope. Bar= $10 \mu \mathrm{m}$. (B) Jurkat cells untreated with the drugs were sub. jected to the phagocytosis assay. Bar $=50 \mu \mathrm{m}$. (C) Nuclear morphology of engulfed Jurkat cells. Jurkat cells treated with doxorubicin were subjected to the phagocytosis assay using FITC-avidin, and the reactive cells were further stained with propidium iodide. Top left, FITC signal from the surface of phagocytosed Jurkat cells; top right, propidium iodide signal from DNA of macrophages and Jurkat cells; bottom left, superimposition of the two signals; bottom right, phase-contrast view. Bar $=50 \mu \mathrm{m}$. (D) Quantification of phagocytosis reactions. Columns and bars are means $+/-\mathrm{SD}$. (E) The phagocytosis asaay was conducted in the presence of Z-VAD-FMK (40 $\mu \mathrm{M})$. The extent of phagocytosis is expressed relative to that of control reactions, taken as 100 . The means of the phagocytic index in control reactions were 15 (DXR) and 26 (BFL). liposomes. Since these two drugs inhibit the growth of cancer cells in different ways, it is likely that anticancer drugs induce PS-mediated phagocytosis of cancer cells irrespective of their mode of action in cell growth inhibi. tion.

PS exists predominantly in the inner leaflet of the plasma membrane bilayer of living cells, and translocates to the outer leaflet upon induction of apoptosis (reviewed in Refs. 29, 30). Our results showed that PS externalization and phagocytosis were both inhibited in the presence of an apoptosis inhibitor. Induction of apoptosis and phagocytosis are thus attributed to the same actions of anticancer drugs, at least for the drugs used in this study. Cell growth inhibition and apoptosis induction by anticancer drugs 
A
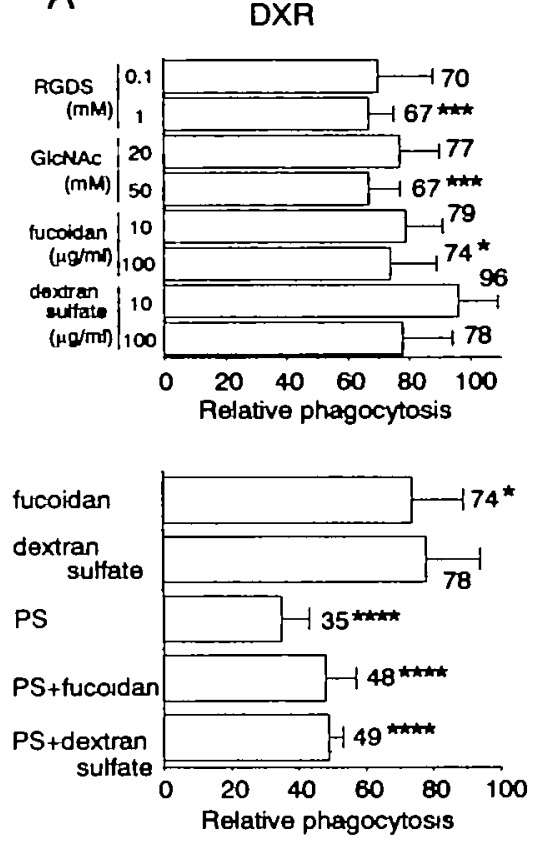
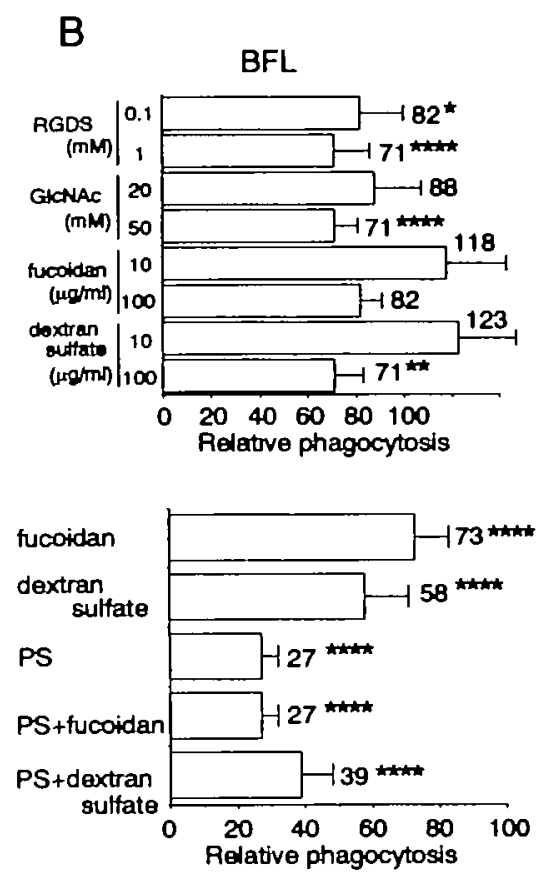

Fig. 5. Effect of potent inhibitors on phagocytosis of anticancer drug-treated Jurkat cells. Various substances were added to the phagocytosis assay. The extent of phagocytosis is shown relative to that with no added inhibitor, taken as 100 . The means of the phagocytic index in control reactions were $17-30$. RGDS, a synthetic peptide containing the integrin. binding sequence RGDS; GlcNAc, $N$-rcetyl-D-glucosamine; fucoidan and dextran sulfate, ligands for class A scavenger receptor; PS, PS-liposomes; PC, PC-liposomes. In the bottom two panels, PS-liposomes $(0.5 \mathrm{mM})$, fucoidan $(100 \mu \mathrm{g} / \mathrm{ml})$, and dextran sulfate $(100 \mu \mathrm{g} / \mathrm{ml})$ were added either alone or in combination. Significance was calculated using Student's $t$ test. " $p<0.05$; " $p<0.02$; ${ }^{* * *} p<0.002 ;{ }^{* * * *} p<0.001$.
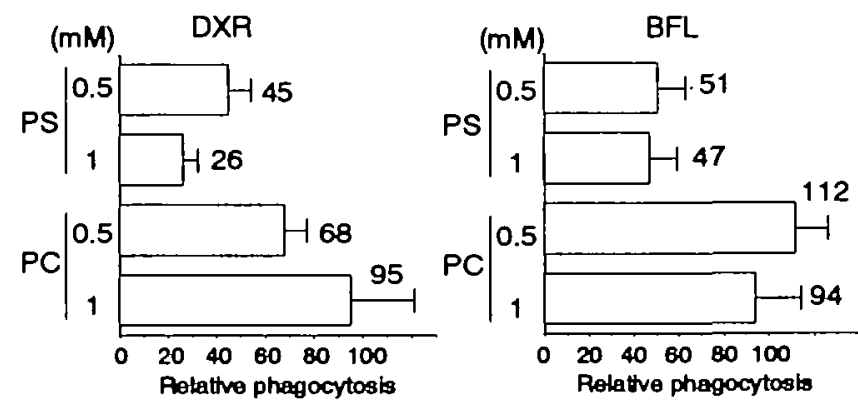

Fig. 4. Effect of liposomes on phagocytosis of anticancer drugtreated Jurkat cells. Phagocytosis reactions were conducted in the presence of PS. or PC-liposomes $(0.5$ or $1 \mathrm{mM})$. The extent of phagocytosis is shown relative to that with no added liposomes, which is taken as 100 . The means of the phagocytic index in control reactions were $22(\mathrm{DXR})$ and 17 (BFL).

might be caused by their distinct activities (31). One could thus modify and improve anticancer drugs simply by removing the former activity, which might cause severe side effects. Moreover, novel drugs may be discovered through screening of chemical and natural products, focusing on apoptosis-inducing activity.

We previously showed that macrophages phagocytose cells that expose PS independently of apoptosis (16). In the present study, drug-treated Jurkat cells were targeted by macrophages before nuclear morphology changed. We speculate that PS externalization by itself, not other apoptotic changes, is sufficient for drug-treated cells to be phagocytosed. It is thus important to elucidate the mechanism by which PS is exposed to the cell surface upon anticancer drug treatment. One suggested mechanism is that changes in the amount and/or activity of enzymes defining localization of the phospholipid lead to its externalization (30). Two enzymes called aminophospholipid translocase (32) and scramblase (33) are believed to control the movement of PS. Characterization of these enzymes is essential for understanding how PS is externalized upon apoptosis induction. Once the precise mechanism becomes clear, novel anticancer drugs that induce cell-surface PS in cancer cells could be developed.

Macrophages probably recognize PS on target cells by using its specific receptor. Several proteins have been identified as candidates for the PS receptor (14,34-41, and reviewed in Ref. 11). Among them, class B scavenger receptor type I and lectin-like oxidized low density lipoprotein receptor (14) were shown to serve as phagocytosisinducing PS receptors in testicular Sertoli cells (40) and vascular endothelial cells (41), respectively. Although the macrophage PS receptor still remains to be identified, one could modify the quantity and/or quality of such a receptor so that macrophages would phagocytose cancer cells with higher selectivity and efficiency. Further studies are needed to understand how anticancer drug-treated cancer cells are recognized and phagocytosed by phagocytic cells, and such studies should provide us with new approaches for the development of novel therapeutic agents against cancer.

We thank K. Nakaya for a kind gift of bufalin, H. Yoshida for her contribution to the initial stage of this atudy, C. Fujii for macrophage preparation, and V. Fadok for critically reading the manuscript.

\section{REFERENCES}

1. Wyllie, A.H., Kerr, J.F.R., and Currie, A.R. (1980) Cell death: the significance of apoptosis. Int. Rev. Cytol 68, 251-306

2. Ellis, R.E., Yuan, J., and Horvitz, H.R. (1991) Mechanisms and functions of cell death. Annu. Rev. Cell Biol. 7, 663-698

3. Albert, M.L., Sauter, B., and Bhardwaj, N. (1998) Dendritic cells acquire antigen from apoptotic cells and induce class l-restricted CTLs. Nature 392, 86-89

4. Rovere, P., Vallinoto, C., Bondanza, A., Crosti, M.C., Rescigno, M., Ricciardi-Castagnoli, P., Rugarli, C., and Manfredi, A.A. (1998) Bystander apoptosis triggers dendritic cell maturation and antigen-presenting function. J. Immunol. 161, 4467-4471 
5. Albert, M.L., Pearce, S.F.A., Francisco, L.M., Sauter, B., Roy, P., Silverstein, R.L., and Bhardwaj, N. (1998) Immature dendritic cells phagocytose apoptotic cells via $\alpha_{\mathrm{v}} \beta_{5}$ and CD36, and cross-present antigens to cytotoxic T lymphocytes. J. Exp. Med. 188, $1359-1368$

6. Inaba, K., Turley, S., Yamaide, F., Iyoda, T., Mahnke, K., Inaba, M., Pack, M., Subklewe, M., Sauter, B., Sheff, D., Albert, M., Bhardwaj, N., Mellman, I., and Steinman, R.M. (1998) Efficient presentation of phagocytosed cellular fragments on the major histocompatibility complex class II products of dendritic cells. $J$. Exp. Med. 188, 2163- 2173

7. Henry, F., Boisteau, O., Bretaudeau, L., Lieubeau, B., Meflah, K., and Grégoire, M. (1999) Antigen-presenting cells that phagocytose apoptotic tumor-derived cells are potent tumor vaccines. Cancer Res. 59, 3329-3332

8. Savill, J., Fadok, V., Henson, P., and Haslett, C. (1993) Phagocyte recognition of cells undergoing apoptosis. Immunol. Today $14,131-136$

9. Savill, J. (1997) Recognition and phagocytosis of cells undergoing apoptosis. Brit. Med. Bull. 53, 491-508

10. Savill, J. (1998) Phagocytic docking without shocking. Nature $392,442-443$

11. Fadok, V.A., Bratton, D.L., Frasch, S.C., Warner, M.L., and Henson, P.M. (1998) The role of phosphatidylserine in recognition of apoptotic cells by phagocytes. Cell Death Differ. 5, 551562

12. Fadok, V.A., Voelker, D.R., Campbell, P.A., Cohen, J.J., Bratton, D.L., and Henson, P.M. (1992) Exposure of phosphatidylserine on the surface of apoptotic lymphocytes triggers specific recognition and removal by macrophages. J. Immunol. $148,2207-2216$

13. Bennett, M.R., Gibson, D.F., Schwartz, S.M., and Tait, J.F. (1995) Binding and phagocytasis of apoptotic vascular smooth muscle cells is mediated in part by exposure of phosphatidylser. ine. Circ. Res. 77, 1136-1142

14. Sawamura, T., Kume, N., Aoyama, T., Moriwaki, H., Hoshikawa, H., Aiba, Y., Tanaka, T., Miwa, S., Kataura, Y., Kita, T., and Masaki, T. (1997) An endothelial receptor for oxidized lowdensity lipoprotein. Nature 386, 73-77

15. Shiratsuchi, A., Umeda, M., Ohbe, Y., and Nakanishi, Y. (1997) Recognition of phosphatidylserine on the surface of apoptotic spermatogenic cells and subsequent phagocytosis by Sertoli cells of the rat. J. Biol. Chem. 272, 2354-2358

16. Shiratsuchi, A., Osada, S., Kanazawa, S., Ohba, Y., and Nakanishi, Y. (1998) Essential role of phosphatidylserine externalization in apoptosing cell phagocytosis by macrophages. Biochem. Biophys. Res. Commun. 246, 549-555

17. Dive, C. and Hickman, J.A. (1991) Drug-target interactions: only the first step in the commitment to a programmed cell death? Br. J. Cancer 84, 192-196

18. Thompson, C.B. (1995) Apoptosis in the pathogenesis and treatment of disease. Science 267, 1456-1462

19. Guchelaar, H.J., Vermes, A., Vermes, I., and Haanen, C. (1997) Apoptosis: molecular mechanism and implications for cancer chemotherapy. Pharm. World Sci 19, 119-125

20. Naito, M., Nagashima, K., Mashima, T., and Tsuruo, T. (1997) Phosphatidylserine externalization is a downstream event of interleukin-1 beta-converting enzyme family protease activation during apoptosis. Blood 89, 2060-2066

21. Fadok, V.A., Savill, J.S., Haslett, C., Bratton, D.L., Doherty, D.E., Campbell, P.A., and Henson, P.M. (1992) Different populations of macrophages use either the vitronectin receptor or the phosphatidylserine receptor to recognize and remove apoptotic cells. J. Immunol 149, 4029-4035

22. Pradhan, D., Krahling, S., Williamson, P., and Schlegel, R.A. (1997) Multiple systems for recognition of apoptotic lymphocytes by macrophages. Mol. Biol. Cell 8, 767-778

23. Koopman, G., Reutelingsperger, C.P.M., Kuijten, G.A.M., Keehnen, R.M.J., Pals, S.T., and van Oers, M.H.J. (1994) Annexin $\mathrm{V}$ for flow cytometric detection of phosphatidylserine expression on B cells undergoing apoptosis. Blood 84, 1415-1420

24. Martin, S.J., Reutelingsperger, C.P.M., and Green, D.R. (1996)
Annexin V: a specific probe for apoptotic cells in Techniques in Apoptosis: A User's Guide (Cotten, Y.G. and Martin, S.J., eds.) pp. 107-119, Portland Press, London

25. Hed, J. (1986) Methods for distinguishing ingested from adhering particles. Methods Enzymol. 132, 198-204

26. Slee, E.A., Zhu, H., Chow, S.C., MacFarlane, M., Nicholson, D.W., and Cohen, G.M. (1996) Benzyloxycarbonyl-Val-Ala-Asp (OMe) fluoromethylketone (Z-VAD.FMK) inhibits apoptosis by blocking the processing of CPP32. Biochem. J. 315, 21-24

27. Spaderi, S., Pedarali, N.G., Focher, F., Montecucco, A., Bordoni, T., Geroni, C., Giuliani, F.C., Ventrella, G., Arcomone, F., and Ciarocchi, G. (1986) DNA polymerases and DNA topoisomerases as targets for the development of anticancer drugs. Anticancer Res. 6, 935-940

28. Zhang, L., Nakaya, K., Yoshida, T., and Kuroiwa, Y. (1992) Induction by bufalin of differentiation of human leukemia cells HL-60, U937 and ML1 toward macrophage/monocyte-like cells and its potent synergistic effect on the differentiation of human leukemia cells in combination with other inducers. Cancer Res. $52,4634-4641$

29. Zachowski, A. (1993) Phospholipids in animal eucaryotic mem branes: transverse asymmetry and movement. Biochem. J. 294, $1-14$

30. Zwaal, R.F.A. and Schroit, A. (1997) Pathophysiologic implications of membrane phospholipid asymmetry in blood cells. Blood 89, 1121-1132

31. Jing, Y., Ohizumi, H., Kawazoe, N., Hashimoto, S., Masuda, Y. Nakajo, S., Yoshida, T., Kuroiwa, Y., and Nakaya, K. (1994) Selective inhibitory effect of bufalin on growth of human tumor cells in vitro: Association with the induction in leukemia HL-60 cells. Jpn. J. Cancer Res. 85, 645-651

32. Tang, X., Halleck, M.S., Schlegel, R.A., and Williamson, P. (1996) A subfamily of P-type ATPase with aminophospholipid transporting activity. Science 272, 1495-1497

33. Zhro, J., Zhou, Q., Wiedmer, T., and Sims, P.J. (1998) Level of expression of phospholipid scramblase regulates induced move. ment of phosphatidylserine to the cell surface. J. Biol. Chem. 273, 6603-6606

34. Savill, J., Hogg, N., Ren, Y., and Haslett, C. (1992) Thrombospondin cooperates with CD36 and the vitronectin receptor in macrophage recognition of neutrophils undergoing apoptosis. $J$. Clin. Invest. 90, 1513-1522

35. Sambrano, G.R. and Steinberg, D. (1995) Recognition of oxidatively damaged and apoptotic cells by an oxidized low density lipoprotein receptor on mouse peritoneal macrophages: Role of membrane phosphatidylserine. Proc. Natl. Acad. Sci. USA 02 1396-1400

36. Platt, N., Suzuki, H., Kurihara, Y., Kodama, T., and Gordon, S. (1996) Role for the class A macrophage scavenger receptor in the phagocytosis of apoptotic thymocytes in vitro. Proc. Natl. Acad. Sci. USA 83, $12456-12460$

37. Fukasawa, M., Adachi, H., Hirota, K., Tsujimoto, M., Arai, H., and Inoue, K. (1996) SRB1, class B scavenger receptor, rocognizes both negatively charged liposomes and apoptotic cells. Exp. Cell Res. 222, 246-250

38. Acton, S., Rigotti, A., Landschulz, K.T., Xu, S., Hobbs, H.H., and Krieger, M. (1996) Identification of scavenger receptor SR-BI as a high density lipoprotein receptor. Science 271, 518520

39. Devitt, A., Moffatt, O.D., Raykundalia, C., Capra, J.D., Simmons, D.L., and Gregory, C.D. (1998) Human CD14 mediates recognition and phagocytosis of apoptotic cells. Nature 392, 505509

40. Shiratsuchi, A., Kawasaki, Y., Ikemoto, M., Arai, H., and Nakanishi, Y. (1999) Role of class B scavenger receptor type I in phagocytosis of apoptotic rat spermatogenic cells by Sertoli cells. J. Biol. Chem. 274, 5901-5908

41. Oka, K., Sawamura, T., Kikuta, K.-I., Itokawa, S., Kume, N., Kita, T., and Masaki, T. (1998) Lectin-like oxidized low-density lipoprotein receptor 1 mediates phagocytosis of aged/apoptotic cells in endothelial cells. Proc. Natl. Acad. Sci. USA 98, 95359540 